"This accepted author manuscript is copyrighted and published by Elsevier. It is posted here by agreement between Elsevier and MTA. The definitive version of the text was subsequently published in [NEUROIMAGE 102(P2): 416-423 (2014), DOI:10.1016/j.neuroimage.2014.08.006]. Available under license CC-BY-NC-ND."

\title{
Authors:
}

\section{Repetition probability effects for inverted faces}

\author{
Mareike Grotheer ${ }^{1,2}$ : mareike.grotheer@uni-jena.de \\ Petra Hermann: hermann.petra@gmail.com \\ Zoltán Vidnyánszky ${ }^{3,5}$ : vidnyanszky.zoltan@gmail.com \\ Gyula Kovács ${ }^{1,2,3}$ : gyula.kovacs@uni-jena.de
}

\section{Author affiliations:}

1 Institute of Psychology, Friedrich-Schiller-University of Jena, Am Steiger 3, 07743 Jena, Germany

2 DFG Research Unit Person Perception, Friedrich-Schiller-University of Jena, Leutragraben 1, 07743 Jena, Germany

3 Department of Cognitive Science, Budapest University of Technology and Economics, Műegyetem rkp. 3, 1111 Budapest, Hungary

4 Faculty of Information Technology and Bionics, Pázmány Péter Catholic University, Práter str. 50/A, 1083 Budapest, Hungary

5 Brain Imaging Centre, Research Centre for Natural Sciences, Hungarian Academy of Sciences, Magyar tudósok körútja 2, 1117 Budapest, Hungary

\section{Corresponding Author:}

Gyula Kovács, Institute of Psychology, Friedrich-Schiller-University of Jena, Am Steiger 3, 07743 Jena, Germany, Tel: +493641/945936, e-mail: gyula.kovacs@uni-jena.de 


\title{
Repetition probability effects for inverted faces
}

\author{
Mareike Grotheer ${ }^{1,2}$, Petra Hermann ${ }^{4}$, Zoltán Vidnyánszky ${ }^{3,5}$, Gyula Kovács ${ }^{1,2,3}$
}

1 Institute of Psychology, Friedrich-Schiller-University of Jena, Jena, Germany; 2 DFG Research Unit Person Perception, Friedrich-Schiller-University of Jena, Jena, Germany; 3 Department of Cognitive Science, Budapest University of Technology and Economics, Budapest, Hungary; 4 Faculty of Information Technology and Bionics, Pázmány Péter Catholic University, Budapest, Hungary; 5 Brain Imaging Centre, Research Centre for Natural Sciences, Hungarian Academy of Sciences, Budapest, Hungary

Corresponding Author: Gyula Kovács, Institute of Psychology, Friedrich-Schiller-University of Jena, Leutragraben 1, 07743 Jena, Germany, Tel: +493641/945936, e-mail: gyula.kovacs@uni-jena.de

\begin{abstract}
It has been shown, that the repetition related reduction of the blood-oxygen level dependent (BOLD) signal is modulated by the probability of repetitions $(\mathrm{P}(\mathrm{rep})$ ) for faces (Summerfield et al., 2008), providing support for the predictive coding (PC) model of visual perception (Rao and Ballard, 1999). However, the stage of face processing where repetition suppression (RS) is modulated by $\mathrm{P}(\mathrm{rep})$ is still unclear. Face inversion is known to interrupt higher level configural/holistic face processing steps and if modulation of RS by $\mathrm{P}(\mathrm{rep})$ takes place at these stages of face processing, $\mathrm{P}(\mathrm{rep})$ effects are expected to be reduced for inverted when compared to upright faces. Therefore, here we aimed at investigating whether $\mathrm{P}$ (rep) effects on RS observed for face stimuli originate at the higher-level configural/holistic stages of face processing by comparing the effect of $\mathrm{P}(\mathrm{rep})$ on $\mathrm{RS}$ for upright and inverted faces. Similarly to previous studies, we manipulated P(rep) for pairs of stimuli in individual blocks of fMRI recordings. This manipulation significantly influenced repetition suppression in the posterior FFA, the OFA and the LO, independently of stimulus orientation. Our results thus reveal that $\mathrm{RS}$ in the ventral visual stream is modulated by $\mathrm{P}(\mathrm{rep})$ even in the case of face inversion and hence strongly compromised configural/holistic face processing. An additional wholebrain analysis could not identify any areas where the modulatory effect of probability was orientation specific either. These findings imply that $\mathrm{P}(\mathrm{rep})$ effects on RS might originate from the earlier stages of face processing.
\end{abstract}


Keywords: repetition suppression, faces, inversion, prediction, expectation

\section{Introduction}

A large body of experiments found, using various electrophysiological and neuroimaging techniques, that the neural activity decreases as a given stimulus is repeated (Gross et al., 1967; Gross et al., 1969; Wiggs and Martin, 1998; Henson and Rugg, 2003; Schacter et al., 2004; Grill-Spector et al., 2006; Krekelberg et al., 2006). The neural mechanisms of this reduced neural activity (denoted as repetition suppression (RS) or adaptation) for repeated stimuli when compared to non-repeated ones are unclear as of today. While one explanation suggests that RS is related to the local alteration of the synaptic inputs/spike frequency of the neurons (Priebe et al., 2002; Kohn and Movshon, 2003; Sawamura et al., 2006; De Baene and Vogels, 2010), the theory of predictive coding (PC) suggests the involvement of top-down connections in determining RS. In the PC approach of visual processing Rao and Ballard (1999) proposed that hierarchically higher areas shape and predict the tuning properties of lower level areas via strong feedback connections. Lower level areas then, in turn, compute the differences (or discrepancies) between the top-down predictions and the actual sensory input. This discrepancy or residual prediction error signal $(\varepsilon)$ is then forwarded to higher areas to re-estimate and update the predictions. The more closely a top-down prediction matches the incoming sensory input, the smaller the feed-forward $\varepsilon$ is, which maximizes the "efficiency" of the CNS in the sense that the neural activity evoked by predicted stimuli is less than that evoked by novel and hence unexpected stimuli (Friston and Kiebel, 2009). According to PC accounts RS reflects the reduction of $\varepsilon$ during subsequent bottom-up/top-down processing iterations within a hierarchical system (Kveraga et al., 2007): repeating a stimulus (or adapting to it) leads to its increased expectation and recalibrates the predictions such that the adapted stimulus evokes reduced $\varepsilon$, which leads to RS in an area.

Indeed, in recent years a large body of direct evidence emerged that supports the PC explanation of RS. The first was Summerfield et al. (2008) who, using functional magnetic resonance imaging (fMRI), showed that the repetition probability $(\mathrm{P}(\mathrm{rep})$ ) of a stimulus determines the degree of RS: the neuroimaging marker of RS, the fMRI adaptation (fMRIa) of the blood oxygenation level-dependent (BOLD) signal, was significantly larger in the fusiform face area (FFA) in blocks with high P(rep) of face stimuli than in blocks where repetitions were less frequent. This suggests that the modulation of $\mathrm{P}(\mathrm{rep})$ allows the subjects to build and modify expectations whether stimulus repetition is likely to occur in a given block or not and that expectation of repetition reduces the BOLD signal further. 
Later, several studies replicated these results for human faces. Larsson and Smith (2012) found that $\mathrm{P}$ (rep) influences fMRIa in several visual areas, provided the subjects attended the face stimuli. Kovács et al. (2012) showed that P(rep) influenced fMRIa equally for overlapping and nonoverlapping peripheral stimulus arrangements in the FFA and the occipital face area (OFA). In an EEG study Summerfield et al. (2011) added the factor of stability/volatility to their previous design in a way that the probabilities of repetition and alternation changed in every 10 (volatile) or only in every 30-40 trials (stable). They found that $\mathrm{P}$ (rep) affected RS only during the stable periods, suggesting that the rate of change of probability of repetitions plays a significant role in determining the magnitude of RS. Egner et al. (2010) used a cueing paradigm to manipulate perceptual expectation while varying stimulus features (faces or houses) and found that the two factors interacted, whereby expectation determined the feature selectivity of FFA, a result that is most efficiently explained by PC models. Altogether, these neuroimaging and electrophysiological studies suggest that the attenuated neural response for repeated stimuli is a direct neural correlate of reduced $\varepsilon$ of the bottom-up and top-down representations, as suggested by the predictive coding models of neural functions (Friston, 2005). Note however, that the role of stimulus expectation in generating RS for non-face stimuli has been directly called into question by some recent studies (Grotheer and Kovács, 2014; Kaliukhovich and Vogels, 2011; Kovács et al., 2013; but for a different conclusion see Mayrhauser et al., 2014). First of all, Kaliukhovich and Vogels (2011), using single-cell recordings in macaque monkeys, found no effect of P(rep) on RS. Similarly, Kovács et al. (2013) showed an effect of stimulus expectation for faces but not for chairs and other every-day objects. Finally, Grotheer and Kovács (2014) found that P(rep) affects RS only for stimuli of expertise. The current study aims at elucidating this unexpected category specificity of the P(rep) effect on fMRIa.

Most theoretical accounts of face perception agree that faces differ from other visual stimuli in their processing in the sense that, as a result of extensive every-day expertise (Bukach et al., 2006), they undergo relatively little decomposition into component parts (Farah et al., 1998). Instead, information across the spatial relations of facial features are integrated into a holistic unit, gestalt or global representation that is more than the combined sum of individual features (Maurer et al., 2002). These configural and/or holistic processes ${ }^{1}$ are specific, higher-level mechanisms of the encoding of upright faces and other categories of high expertise (Gauthier and Tarr, 2002; Rossion, 2008).

\footnotetext{
${ }^{1}$ Please note that the terms holistic and configural processing are sometimes used interchangeably in the literature. Here, following the terminology of Tanaka and Gordon (2011), we refer to both those processing steps that require the estimation of metric distances (configural) or the integration of featural/configural information of a face into a global percept (holistic; Sergent, 1984).
} 
Weather earlier steps, responsible for the structural description of facial features or relatively later, configural/holistic stages of face processing are affected by $\mathrm{P}(\mathrm{rep})$ effects is unclear as of today. One possibility is that the extensive experience we have with faces leads to enhanced configural/holistic processing of stimuli (Bukach et al., 2006), which, in turn, gives rise to the P(rep) effects. In other words, theoretically it is possible that only the configural/holistic processing stages, which are specific for faces are affected by $\mathrm{P}(\mathrm{rep})$ and that this explains the face specificity of probability effects (Kaliukhovich and Vogels, 2011; Kovács et al., 2013).

Experimentally, configural/holistic processing is measured by tasks such as the whole-part task, showing the superiority of wholes over parts (Tanaka and Farah, 1993), the composite task (Young et al., 1987) and the face inversion effect (FIE; Yin, 1969). FIE is an observation that a 180-degree in-plane rotation impairs recognition significantly more for faces than for non-face objects. Most current studies suggest that since inversion preserves the low-level features of faces, the FIE is attributed to the interruption of higher-level processes such as the configural processing of spatial relationships of object parts as well as the face specific holistic processing (Rossion and Gauthier, 2002; Rossion, 2008; but see Yovel and Kanwisher, 2004 for another conclusion). Therefore, it seems that inversion leads to qualitatively different processing of stimuli belonging to categories of high expertise, such as faces. In line with this specificity, recent behavioral electrophysiological and neuroimaging results suggest that the visual system processes inverted faces more similarly to nonface objects than to upright faces (Haxby et al., 1999; Rossion et al., 2000; Rosburg et al., 2010; Kloth et al., 2013).

Therefore, we reasoned that if the face-specificity of $\mathrm{P}(\mathrm{rep})$ effects is due to the unique (holistic/configural) processing steps of an upright face then stimulus inversion, a manipulation that interrupts these processing steps, should interfere with the modulatory effects of $\mathrm{P}(\mathrm{rep})$ as well. However, if inversion does not affect $\mathrm{P}$ (rep) modulations of RS, the face-specificity of this effect can't be explained by the holistic/configural face processing steps.

\section{Materials and Methods}

\subsection{Subjects}

Thirty healthy volunteers (8 male; 2 left-handed; mean age $( \pm S D): 22.8$ (3.2) years) participated in the experiment. All subjects had normal or corrected to normal vision and gave informed written consent in accordance with the protocols approved by the Ethical Committee of the FriedrichSchiller-University of Jena. One subject had to be excluded from the study due to technical difficulties in the acquisition of the data, while in three subjects the regions of interests could not be 
identified reliably. Therefore the present results are based on the data of 26 subjects.

\subsection{Stimulation and Procedure}

The experimental design of the current study was similar to that of Summerfield et al. (2008) and to that of Kovács et al. (2012), with the exception that the face stimuli were presented in two different orientations (Fig 1A). 240 gray-scale, digital photos of full-frontal Caucasian faces, similar to the face stimuli of (Kovács et al., 2012; Kovács et al., 2013), were fit behind a circular mask (diameter=5.5²) and either presented upright (UPR), or inverted (INV) in different runs of fMRI recordings. No stimulus occurred in more than one trial during each run. Stimuli were placed in the center of the screen on a uniform grey background. They were presented for $250 \mathrm{~ms}$ each pairwise, separated by an inter-stimulus interval that varied between 400 and $600 \mathrm{~ms}$ and followed randomly by a 1 or $2 \mathrm{sec}$ long inter-trial interval. The first stimulus (S1), was either identical to (Repetition Trial, RepT) or different from the second stimulus (S2) (Alternation Trial, AltT). All stimuli were presented with $2.75^{\circ}$ visual angle in non-target trials, target stimuli were reduced in size by $54 \%$. To reduce local feature adaptation the size of either S1 or S2 (chosen randomly) was reduced by $18 \%$. Stimuli were back-projected via an LCD video projector (NEC GT 1150, NEC Deutschland GmbH, Ismaning, Germany, with modified lens for short focal point) onto a translucent circular screen, placed inside the scanner bore. Presentation was controlled via Matlab R2013a (The MathWorks, Natick, MA, USA), using Psychtoolbox (Version 3.0.9). In addition to the different trial types, two different types of blocks were presented to the subjects (Fig 1B). In the Repetition Blocks (RepB) 75\% of the nontarget trials were RepT while 25\% were AltT. In the Alternation Blocks (AltB) 75\% of the non-target trials were Alt'T and 25\% were RepT. With the exception of the first four trials of each block, which always consisted out of the more frequent trial type of that specific block (RepT in RepB and Alt $T$ in AltB), Rep'T and AltT were presented randomly within the blocks. In addition, 20\% of all trials were target trials, whereas target trials could be Alt $\mathrm{T}$ or RepT with the same relative probability. Hence, overall, Alternation Blocks contained 70\% AltT and 30\% RepT and Repetition Blocks consisted of 70\% RepT and 30\% AltT (identical to the paradigm of Summerfield et al., 2008). Both Alternation and Repetition Blocks contained 20 trials and were repeated 4 times during each run, so that a run contained 160 trials. The different blocks were separated from each other by a 7 sec pause during which the phrase "Short Break" was presented centrally together with a countdown. The participants" task was to maintain central fixation throughout the trials and to signal the occurrence of target stimuli by pressing a button. Four runs were presented in total, whereas the order of upright and upside-down runs was counterbalanced across subjects. 
-- Figure 1 about here --

\subsection{Imaging Parameters and Data Analysis}

A 3-Tesla MR scanner (Siemens Magnetom Trio, Erlangen, Germany) was used to perform the study. During the functional blocks we continuously acquired images (34 slices, $10^{\circ}$ tilted relative to axial, T2* weighted EPI sequence, TR $=2000 \mathrm{~ms}$; TE $=30 \mathrm{~ms}$; flip angle $=90^{\circ} ; 64$ x 64 matrices; inplane resolution: $3 \times 3 \mathrm{~mm}$; slice thickness: $3 \mathrm{~mm} ; 20 \%$ inter-slice interval). To additionally obtain a 3D structural scan, high-resolution sagittal T1-weighted images were acquired using a magnetization EPI sequence (MP-RAGE; TR $=2300 \mathrm{~ms}$; TE $=3.03 \mathrm{~ms} ; 1 \mathrm{~mm}$ isotropic voxel size). Details of preprocessing and statistical analysis are described elsewhere (Cziraki et al., 2010). Briefly, the functional images were realigned, normalized to the MNI-152 space, resampled to $2 \times 2 \times 2 \mathrm{~mm}$ resolution and spatially smoothed with a Gaussian kernel of $8 \mathrm{~mm}$ FWHM (SPM8, Welcome Department of Imaging Neuroscience, London, UK). Separate functional localizer runs (640 sec long, $20 \mathrm{sec}$ epochs of faces, objects, human bodies and Fourier randomized versions of faces, interleaved with $20 \mathrm{sec}$ of blank periods, $2 \mathrm{~Hz}$ stimulus repetition rate; $300 \mathrm{~ms}$ exposition time; 200 ms blank) were used to determine Regions of Interest (ROIs), which were analyzed using the MARSBAR 0.42 (Brett et al., 2002) toolbox for SPM. As the anterior part of the FFA, also called mFUS (Weiner and Grill-Spector, 2010, 2013) was found in the right hemisphere only in 8 and in the left hemisphere only in 11 subjects, we present these results in the Supplementary Material only and concentrated our main analyses on the more posterior part of the FFA (pFFA or pFUS, Weiner and Grill-Spector, 2010, 2013). The pFFA [n=25; average MNI coordinates ( \pm SE): $-41( \pm 0.9),-63( \pm 1.4)$, $-18( \pm 1.0)$ and $42( \pm 0.9),-63( \pm 1.4),-17( \pm 1.1)$; average ROI size $( \pm S E): 34( \pm 2)$ and $37( \pm 5)$ voxels; for the left and right hemispheres, respectively] was determined individually as an area responding more intensely to faces than to objects and to Fourier randomized versions of faces $\left(\mathrm{p}<0.001_{\text {UnCORRECTED }}\right)$. The OFA [ $\mathrm{n}=26$; average MNI coordinates $( \pm \mathrm{SE}):-43( \pm 1.1),-83( \pm 1.2),-3$ $( \pm 1.5)$ and $44( \pm 1.1),-83( \pm 1.6),-2( \pm 1.3)$; average ROI size ( \pm SE): $42( \pm 9)$ and $37( \pm 5)$ voxels; for the left and right hemispheres, respectively] was determined individually as an area responding more intensely to faces than to Fourier randomized versions of faces $\left(p<0.001_{\text {UNCORRECTED }}\right)$ and the LO [n=26; average MNI coordinates ( \pm SE): -40 ( \pm 0.8$),-85( \pm 1.4),-14( \pm 1.0)$ and $40( \pm 0.9),-83( \pm 1.3),-$ 10 ( \pm 1.1$)$; average ROI size ( \pm SE): $97( \pm 28)$ and $91( \pm 14)$ voxels; for the left and right hemispheres, respectively] was determined as an area responding more intensely to objects than to Fourier 
$\left(\mathrm{p}<0.001_{\text {UNCORRECTED }}\right)$. The average locations of these ROIs are presented together with localizer whole-brain results in Fig 2. A time series of the mean voxel value within the areas of interest was calculated and extracted from our event-related sessions. The 8 experimental conditions (AltB_AltT, AltB_RepT, RepB_AltT, RepB_RepT, each in the two different orientations) as well as the target trials were defined as separated regressors, which were convoluted with the canonical Haemodynamic Response Function (HRF) of SPM8, for a General Linear Model (GLM) analysis of the data. We performed repeated measures ANOVAs for each area separately with stimulus orientation (2), hemisphere (2), block (2) and trial (2) as factors. Post-hoc analyses were performed using Fisher LSD tests.

To compare the magnitude of repetition suppression of UPR and INV conditions directly we performed the following additional analysis. First we calculated a repetition suppression index score (RSI) following the methods of (Axelrod and Yovel, 2011; Kaliukhovich and Vogels, 2011) using the equation $\mathrm{RSI}=\left(\mathrm{R}_{\text {alt }}-\mathrm{R}_{\text {rep }}\right) /\left(\left|\mathrm{R}_{\text {alt }}\right|+\left|\mathrm{R}_{\text {rep }}\right|\right)$, where $\mathrm{R}_{\text {alt }}$ and $\mathrm{R}_{\text {rep }}$ are the average responses in the Alt $\mathrm{T}$ and RepT within a given block and orientation condition, respectively. Positive values indicate more pronounced responses in the Alt'T than in the RepT, negative values indicate the opposite and zero values indicate the absence of any response differences between the two trial types (Kovács et al., 2012).

-- Figure 2 about here --

\section{Results}

\subsection{Performance}

The subjects detected the target stimuli for the UPR and INV conditions on average with 80.1 and $82.9 \%\left(4.3\right.$ and $4.00 \% \pm \mathrm{SE}$ ) accuracy (statistically not different $\left(\mathrm{F}(1,25)=3.4, \mathrm{p}=0.14, \eta_{\mathrm{p}}{ }^{2}=0.1\right)$, respectively. During informal questioning at the end of the experiment none of the subjects reported awareness of the different repetition probabilities in the presented blocks.

\subsection{ROI analyses}

\subsubsection{Posterior FFA}

We observed larger BOLD responses in the right, when compared to the left pFFA (main effect of hemisphere: $\left.\mathrm{F}(1,24)=24.93, \mathrm{p}=0.00004, \eta_{\mathrm{p}}{ }^{2}=0.50\right)$, but this effect was not in interaction with any other factors. A significant RS was found in the pFFA (Fig 3A; main effect of trial type: 
$\left.\mathrm{F}(1,24)=7.97, \mathrm{p}=0.009, \eta_{\mathrm{p}}{ }^{2}=0.25\right)$ in the form of larger BOLD responses for Alt $\mathrm{T}$ when compared to RepT, supporting previous results. The magnitude of the RS was more pronounced in the RepB as compared to the AltB as suggested by the block type $x$ trial type interaction $(F(1,24)=9.68, p=0.005$, $\left.\eta_{\mathrm{p}}{ }^{2}=0.29\right)$. This was largely due to the fact that AltT and RepT led to similar responses in AltB (posthoc test for AltT vs RepT: $\mathrm{p}=0.76$ and $\mathrm{p}=0.35$ for UPR and INV, respectively), while AltT was significantly larger than RepT in $\operatorname{RepB}(\mathrm{p}=0.05$ and $\mathrm{p}=0.02$ for UPR and INV, respectively). In addition, the effect of block was not significant $\left(F(1,24)=2.06, \mathrm{p}=0.16, \eta_{\mathrm{p}}{ }^{2}=0.08\right)$, although Alt $\mathrm{T}$ led to greater responses in the RepB than in the AltB for the UPR stimuli $(p=0.02)$, alike to the findings of our previous publication (Kovács et al., 2012). Most important to our aims, the interaction of block type and trial type was not significantly different for UPR and INV faces (orientation $\mathrm{x}$ block $\mathrm{x}$ trial interaction: $\left.\mathrm{F}(1,24)=0.35, \mathrm{p}=0.56, \eta_{\mathrm{p}}{ }^{2}=0.01\right)$. This suggests similar modulatory effects of $\mathrm{P}(\mathrm{rep})$ on repetition suppression, independently of the orientation of the face stimulus.

In accordance with these results, the RSI (Fig 3B) was significantly larger for RepB when compared to AltB (main effect of block: $\mathrm{F}(1,24)=7.39, \mathrm{p}=0.01, \eta_{\mathrm{p}}{ }^{2}=0.23$ ). The direct comparisons of the RSIs in the UPR and INV conditions revealed neither a significant main effect of orientation $\left(\mathrm{F}(1,24)=0.09, \mathrm{p}=0.78, \eta_{\mathrm{p}}{ }^{2}=0.004\right)$ nor a significant interaction of block type and orientation $\left(\mathrm{F}(1,24)=0.64, \mathrm{p}=0.43, \eta_{\mathrm{p}}{ }^{2}=0.03\right)$. These results support further the conclusion that the observed repetition suppression in the pFFA is independent of the orientation of the stimulus.

-- Figure 3 about here --

\subsubsection{OFA}

As in the pFFA, the probability of face repetition influenced repetition suppression significantly in the OFA (Fig 4A) as well (block x trial interaction: $\left(F(1,25)=6.39, \mathrm{p}=0.02, \eta_{\mathrm{p}}{ }^{2}=0.20\right)$, which was due to the fact that significant RS was observed in RepB but not in AltB (post-hoc test for Alt'T vs RepT: $\mathrm{p}=0.02$ and $\mathrm{p}=0.28$ for $\mathrm{RepB}$ and $\mathrm{AltB}$, respectively). There was no main effect of hemisphere $\left(\mathrm{F}(1,25)=1.41, \mathrm{p}=0.25, \eta_{\mathrm{p}}{ }^{2}=0.05\right)$ or block $\left(\mathrm{F}(1,25)=0.44, \mathrm{p}=0.51, \eta_{\mathrm{p}}{ }^{2}=0.02\right)$, even though post-hoc test indicated an increased bold response to Alt $T$ in $\operatorname{RepB}$ when compared to AltB ( $\mathrm{p}=0.06)$. Most importantly, a marginally significant effect of orientation was observed in the $\operatorname{OFA}(F(1,25)=3.76$, $\left.\mathrm{p}=0.06, \eta_{\mathrm{p}}{ }^{2}=0.13\right)$, which, however, did not influence $\mathrm{P}(\mathrm{rep})$ modulatory effects $(\mathrm{F}(1,25)=1.13$, $\left.\mathrm{p}=0.72, \eta_{\mathrm{p}}^{2}=0.05\right)$ thereby further supporting their independence. 


\subsubsection{LO}

In the LO we observed a main effect of hemisphere $\left(F(1,25)=23.18, p=0.00006, \eta_{p}{ }^{2}=0.48\right)$ in the sense that the responses were larger for the right when compared to left hemisphere, but this effect did not interact with any other factors. Although AltT led to greater responses in RepB than in AltB (post-hoc test: $\mathrm{p}=0.0001)$, the main effect of block was not significant $(\mathrm{F}(1,25)=3.70, \mathrm{p}=0.07$, $\left.\eta_{\mathrm{p}}{ }^{2}=0.13\right)$. In contrast to $\mathrm{pFFA}$ and OFA, LO presented a main effect of stimulus orientation $\left(\mathrm{F}(1,25)=7.92, \mathrm{p}=0.009, \eta_{\mathrm{p}}{ }^{2}=0.24\right)$ as bold responses were significantly larger for INV when compared to UPR. This indicates the LO as a potential source of the face inversion effects. However, the effect of orientation did not interact with any other factors. We found P(rep) modulatory effects in LO (Fig 4B), as indicated by the block $x$ trial interaction $(F(1,25)=16.23$, $\left.\mathrm{p}=0.0005, \eta_{\mathrm{p}}{ }^{2}=0.39\right)$. This effect was due to the fact that significant RS was observed in the RepB $(\mathrm{p}=0.001$ for Alt $\mathrm{T}$ vs RepT) but not in the AltB $(\mathrm{p}=0.054)$. The block $\mathrm{x}$ trial interaction was independent of stimulus orientation (3-way interaction stimulus*block*trial: $F(1,25)=0.03, p=0.85$, $\eta_{\mathrm{p}}{ }^{2}=0.001$ ), suggesting similar effects for UPR and INV faces. The concurrent existence of a main effect of stimulus and $\mathrm{P}(\mathrm{rep})$ effect without any interaction strongly suggest the independence of the two effects.

---Figure 4 about here----

Altogether, our ROI analyses confirmed previous findings emphasizing the role of $\mathrm{P}(\mathrm{rep})$ in determining the magnitude of repetition suppression for faces and extended these results by showing that the modulatory effect of $\mathrm{P}(\mathrm{rep})$ is independent of the orientation of the face stimuli.

\subsection{Whole-brain analyses}

\subsubsection{Orientation:}

It is theoretically possible that the neural processing of UPR and INV faces differs outside the areas of the core face-processing network, tested by the ROI based analysis of the current study. Therefore, first we performed a second-level whole-brain analysis for the UPR vs INV contrast. This indicated no significant activations with the commonly applied rigorous threshold of $\mathrm{p}_{\mathrm{FWE}}<0.05$ (cluster extent of $>50$ ). However, in order to ensure that no activations were overlooked, we also applied a more liberal threshold $\left(\mathrm{p}<0.0001_{\text {UNCORRECTED }}\right.$; cluster extent of $>20$ voxels) and found one 
cluster of activation in the right cingulate gyrus (Fig 5A; MNI coordinates: 22, 36, 12; cluster size: 32 voxels). We used this average cluster of activation as an additional ROI to test for differences in $\mathrm{P}$ (rep) effects on RS on a subject-by-subject basis, but found neither a main effect of trial $\left(\mathrm{F}(1,25)=2.25, \mathrm{p}=0.15, \eta_{\mathrm{p}}{ }^{2}=0.08 ;(1,25)=1,24, \mathrm{p}=0.28, \eta_{\mathrm{p}}{ }^{2}=0.05\right.$; for UPR and INV respectively), nor a main effect of block $\left(F(1,25)=0.14, \mathrm{p}=0.71, \eta_{\mathrm{p}}{ }^{2}=0.005 ; \mathrm{F}(1,25)=0.35, \mathrm{p}=0.56, \eta_{\mathrm{p}}{ }^{2}=0.01\right.$; for UPR and INV respectively), and no significant interaction of block and trial for neither upright faces $\left(\mathrm{F}(1,25)=0.44, \mathrm{p}=0.51, \eta_{\mathrm{p}}{ }^{2}=0.02\right)$ nor inverted faces $\left(\mathrm{F}(1,25)=1.17, \mathrm{p}=0.29, \eta_{\mathrm{p}}{ }^{2}=0.04\right)$. These results suggest the involvement of the area in configural/holistic face processing without any RS or predictive effects. However, these conclusions should be considered with caution as the location of this ROI was derived from the same data set as it was tested on (for the issue of "double-dipping" see Kriegeskorte et al., 2009). The opposite contrast (INV vs UPR) led to significant clusters of activation in the LO as well as in the left postcentral gyrus (Fig 5A; MNI coordinates: 36, -90, 4; -36, -92, 2; -44, -26, 34; cluster sizes: 194; 227; 83; threshold: $\mathrm{p}<0.0001_{\text {UNCORRECTED }}$; cluster extent of $>20$ voxels), supporting the main effect of orientation, observed in our ROI based analysis as described above.

\subsubsection{Repetition probability effects}

Next, to test whether other areas possibly reflect the repetition probability modulation effects as well, we also performed whole-brain second-level random-effects analyses separately for the UPR and INV stimuli. Testing the main effect of trial type (Alt'T vs Rep'T) and the main effect of block type (AltB vs RepB) led to no significant activations in any additional brain regions even with the liberal threshold of $\mathrm{p}<0.0001_{\text {UNCORRECTED }}$ (cluster extent $>20$ ) for either stimulus orientations. The contrast testing the interaction of trial and block types [(AltB_AltT vs AltB_RepT) vs (RepB_AltT vs RepB_RepT)], i.e. the P(rep) effect on RS, showed significant activations in the lingual gyrus, corresponding to the early visual areas for UPR (Fig 5B, left; MNI coordinates: $-12,-78,-2 ;-10,-88$, 18; cluster sizes: 63; 90; threshold: $\mathrm{p}_{\mathrm{FWE}}<0.05$ cluster extent of $>50$ ) and with a more liberal threshold also for INV (Fig 5B; MNI coordinates: 14, -80, -10; cluster size: 33; threshold: $\mathrm{p}<0.0001_{\text {UNCORRECTED }}$; cluster extent of $>20$ voxels). The same threshold identified an additional cluster of activations in the superior temporal gyrus, corresponding to the anterior STS (Fig 5B, middle), for upright faces (MNI coordinates: 56, -16, -2; -60, -44, 4; cluster sizes: 237; 326; threshold: $\mathrm{p}<0.0001_{\text {UNCORRECTED }}$; cluster extent of $>20$ voxels) but not for inverted faces (Fig 5B, right).

\subsubsection{Orientation specific probability effects:}


To test explicitly the orientation dependence of the observed P(rep) effect in STS we performed an additional ROI analyses using this average cluster on a subject-by-subject basis. We found significant interaction of block and trial $\left(\mathrm{F}(1,25)=36.12, \mathrm{p}=0.000003, \eta_{\mathrm{p}}{ }^{2}=0.59\right)$ without a main effect of trial $\left(\mathrm{F}(1,25)=0.18, \mathrm{p}=0.67, \eta_{\mathrm{p}}{ }^{2}=0.007\right)$ or block $\left(\mathrm{F}(1,25)=0.22, \mathrm{p}=0.64, \eta_{\mathrm{p}}{ }^{2}=0.009\right)$. Most importantly, neither the main effect of stimulus orientation nor any of its interactions were significant $(p>0.1$ for each comparison). To further confirm that no other cortical area shows orientation specific P(rep) effects on RS we performed a whole-brain group analysis testing the three-way interaction of orientation x block $\mathrm{x}$ trial as well (following the method by Henson and Penny, 2003). This analysis revealed no significant clusters even with a very liberal threshold $(\mathrm{p}<0.001$, cluster extend 20 voxels). Overall our results hence suggest that repetition modulation effects are independent of holistic/configural processing.

\section{Discussion}

We found that repetition probability affects the magnitude of repetition suppression in the pFFA, the OFA and the LO for upright and inverted faces similarly. As face inversion leads to the interruption of configural/holistic steps of face processing, our results suggest that $\mathrm{P}(\mathrm{rep})$ effects on RS might originate either from the early structural or from later, post-configural stages of face processing.

Most theories of face perception agree that upright faces are processed qualitatively differently from inverted faces, although the exact nature of this difference is currently under heavy debate (for reviews see Rossion and Gauthier, 2002; Tanaka and Gordon, 2011). Several studies suggested that face inversion disturbs the processing of configural/holistic information in a face more than that of the facial features either overall across the entire face (Rhodes et al., 1993; Cabeza and Kato, 2000; Freire et al., 2000; Leder and Bruce, 2000; Barton et al., 2001; Leder et al., 2001; Rossion and Gauthier, 2002; Leder and Carbon, 2006) or at least in the lower, mouth region (Xu and Tanaka, 2013; Tanaka et al, 2009). Other studies, however, emphasize that the processing of featural and configural information are equally disrupted by face inversion (Riesenhuber et al., 2004; Yovel and Kanwisher, 2004; for a review see Tanaka and Gordon, 2011). Despite the lack of consensus regarding the details ${ }^{2}$, common to all these studies is the assumption that certain mechanisms, exclusively involved in the processing of upright faces are interrupted by picture-plane inversion.

\footnotetext{
${ }^{2}$ Note that the current study was not designed to explicitly investigate if the processing of features, configurations or both are disrupted by picture-plane inversion and that a detailed discussion of the issue is beyond the scope of the present article.
} 
Recent results extend this notion further and suggest that the processing of inverted faces is more similar to the processing of non-face objects than to that of upright faces (Haxby et al., 1999; Rossion et al., 2000; Rosburg et al., 2010; Kloth et al., 2013), presumably due to the enhanced functional connectivity of the face specific areas with higher-order object sensitive areas (Nguyen et al., 2013). These findings could also explain the increased bold response to inverted as opposed to upright faces, which we observed in the object sensitive LO.

Interestingly, both the configural and the joint feature + configural accounts of the face inversion effect suggest the FFA as the cortical area where the differential processing of UPR and INV faces occurs (Yovel and Kanwisher, 2004, 2005; Mazard et al., 2006; Maurer et al., 2007; Rhodes et al., 2009). The fact, however, that the magnitude of RS was similar for UPR and INV faces in the current experiment argues against the FFA being sensitive to picture-plain inversion. Our results rather support those of a recent study (James et al., 2013) that showed, using multiple combinations of features with and without the context of a whole face, that the FFA is unlikely to be the site of the face specific processes that are responsible for producing the FIE. On the other hand, other studies found larger fMRIa for UPR when compared to INV faces (Mazard et al., 2006; Schiltz and Rossion, 2006) in the FFA. The conflicting results might be explained by the fact that previous studies used a block design and several repetitions of the images while our current event-related design involved the presentation of pairs of stimuli. Further research is required to ultimately elucidate whether or not the FFA is sensitive to face inversion.

Predictive coding is one of the universal theories of visual information processing. It postulates that vision is a hierarchical process in which higher order areas shape and predict the tuning properties of lower level areas via strong feedback connections (Friston, 2005). This is achieved by suppressing the repeated or predicted, and hence redundant, neural responses in lower level areas that are consistent with the higher level expectations, resulting in a suppressed response of these neuronal populations and in an efficient encoding mechanism (Friston and Kiebel, 2009).

Given the universal nature of PC models it is surprising that some recent results are at odds with the PC explanations of repetition related response suppressions of cortical neurons. Two studies, using non-face stimuli, found no evidences of repetition probability modulations of RS in the macaque inferior temporal cortex (Kaliukhovich and Vogels, 2011) and in the human lateral occipital cortex (Kovács et al., 2013). These results with natural objects, fractals and chairs as stimuli are in strong contrast with those studies which found a significant modulation of RS by $\mathrm{P}(\mathrm{rep})$ for faces (Summerfield et al., 2008; Kovács et al., 2012; Larsson and Smith, 2012) as well as for voices (Andics 
et al., 2013). Indeed, recently, Grotheer and Kovács (2014) could demonstrate P(rep) effects for roman letters, another object category with which literate humans have great experience and which shows inversion effects in expert subjects (Bruyer, and Crispeels, 1992). Their study was, however, not designed to test the underlying mechanisms by which expertise might facilitate $\mathrm{P}(\mathrm{rep})$ effects. One possibility is that extensive experience leads to enhanced holistic/configural processing of stimuli (Bukach et al., 2006), which, in turn, gives rise to the P(rep) effects for faces. However, the results of the current study make this explanation unlikely, as similar $\mathrm{P}$ (rep) effects on RS were observed for upright and inverted faces. The fact that we observed P(rep) modulations in the early visual areas, LO, OFA, STS and posterior FFA for both upright and inverted faces supports the results of Larsson and Smith (2012) who showed P(rep) effects throughout the ventral visual cortex for upright faces and extends those results by showing that $\mathrm{P}$ (rep) influences RS for upright and inverted faces in these areas similarly.

In summary, we observed an effect of contextual information on repetition suppression for faces in the ventral visual stream, which was independent of the orientation of the stimuli. These findings suggest that the specific configural/holistic processing steps of faces do not affect the probability modulation of repetition suppression.

Acknowledgement: Supported by a Deutsche Forschungsgemeinschaft Grant (KO 3918/1-2; 2-1).

\section{References}

Aguirre GK, Singh R, D'Esposito M (1999) Stimulus inversion and the responses of face and object-sensitive cortical areas. Neuroreport 10:189-194.

Andics A, Gál V, Vicsi K, Rudas G, Vidnyánszky Z (2013) FMRI repetition suppression for voices is modulated by stimulus expectations. NeuroImage 69:277-83.

Axelrod V, Yovel G (2011) Nonpreferred stimuli modify the representation of faces in the fusiform face area. J Cogn Neurosci 23:746-756.

Barton JJ, Keenan JP, Bass T (2001) Discrimination of spatial relations and features in faces: Effects of inversion and viewing duration. Br J Psychol 92 Part 3:527-549.

Brett M, Johnsrude IS, Owen AM (2002) The problem of functional localization in the human brain. Nat Rev Neurosci 3:243-249.

Bruyer R, Crispeels G (1992) Expertise in person recognition. Bull. Psychon. Soc. 30:501-504.

Bukach CM, Gauthier I, Tarr MJ (2006) Beyond faces and modularity: the power of an expertise framework. Trends Cogn Sci 10:159-166.

Cabeza R, Kato T (2000) Features are also important: contributions of featural and configural processing to face recognition. Psychol Sci 11:429-433.

Cziraki C, Greenlee MW, Kovacs G (2010) Neural correlates of high-level adaptation-related aftereffects. J Neurophysiol 103:1410-1417.

De Baene W, Vogels R (2010) Effects of adaptation on the stimulus selectivity of macaque inferior temporal spiking activity and local field potentials. Cereb Cortex 20:2145-2165. 
Egner T, Monti JM, Summerfield C (2010) Expectation and surprise determine neural population responses in the ventral visual stream. J Neurosci 30:16601-16608.

Farah MJ, Wilson KD, Drain M, Tanaka JN (1998) What is "special" about face perception? Psychol Rev 105:482-498.

Freire A, Lee K, Symons LA (2000) The face-inversion effect as a deficit in the encoding of configural information: direct evidence. Perception 29:159-170.

Friston K (2005) A theory of cortical responses. Philos Trans R Soc Lond B Biol Sci 360:815-836.

Friston K, Kiebel S (2009) Predictive coding under the free-energy principle. Philos Trans R Soc Lond B Biol Sci 364:1211-1221.

Gauthier I, Tarr MJ (2002) Unraveling mechanisms for expert object recognition: bridging brain activity and behavior. J Exp Psychol Hum Percept Perform 28:431-446.

Grill-Spector K, Henson R, Martin A (2006) Repetition and the brain: neural models of stimulus-specific effects. Trends Cogn Sci 10:14-23.

Grotheer M, Kovács G (2014) Repetition probability effects depend on prior experiences. J Neurosci 34:66406646.

Gross CG, Bender DB, Rocha-Miranda CE (1969) Visual receptive fields of neurons in inferotemporal cortex of the monkey. Science 166:1303-1306.

Gross CG, Schiller PH, Wells C, Gerstein GL (1967) Single-unit activity in temporal association cortex of the monkey. J Neurophysiol 30:833-843.

Haxby JV, Ungerleider LG, Clark VP, Schouten JL, Hoffman EA, Martin A (1999) The effect of face inversion on activity in human neural systems for face and object perception. Neuron 22:189-199.

Henson RNA, Penny WD (2003) ANOVAs and SPM. Technical report, Wellcome Department of Imaging Neuroscience.

Henson RNA, Rugg MD (2003) Neural response suppression, haemodynamic repetition effects, and behavioural priming. Neuropsychologia 41:263-270.

James TW, Arcurio LR, Gold JM (2013) Inversion effects in face-selective cortex with combinations of face parts. J Cogn Neurosci 25:455-464.

Kaliukhovich DA, Vogels R (2011) Stimulus repetition probability does not affect repetition suppression in macaque inferior temporal cortex. Cereb Cortex 21:1547-1558.

Kanwisher N, Tong F, Nakayama K (1998) The effect of face inversion on the human fusiform face area. Cognition 68:B1-11.

Kloth N, Itier RJ, Schweinberger SR (2013) Combined effects of inversion and feature removal on N170 responses elicited by faces and car fronts. Brain Cogn 81:321-328.

Kohn A, Movshon JA (2003) Neuronal adaptation to visual motion in area MT of the macaque. Neuron 39:681-691.

Kovács G, Iffland L, Vidnyanszky Z, Greenlee MW (2012) Stimulus repetition probability effects on repetition suppression are position invariant for faces. Neuroimage 60:2128-2135.

Kovács G, Kaiser D, Kaliukhovich DA, Vidnyanszky Z, Vogels R (2013) Repetition probability does not affect fMRI repetition suppression for objects. J Neurosci 33:9805-9812.

Krekelberg B, Boynton GM, van Wezel RJ (2006) Adaptation: from single cells to BOLD signals. Trends Neurosci 29:250-256.

Kriegeskorte N, Simmons WK, Bellgowan PS, Baker CI (2009) Circular analysis in systems neuroscience: the dangers of double dipping. Nat Neurosci 12:535-540.

Kveraga K, Ghuman AS, Bar M (2007) Top-down predictions in the cognitive brain. Brain Cogn 65:145-168.

Larsson J, Smith AT (2012) fMRI repetition suppression: neuronal adaptation or stimulus expectation? Cereb Cortex 22:567-576.

Leder H, Bruce V (2000) When inverted faces are recognized: the role of configural information in face recognition. Q J Exp Psychol A 53:513-536.

Leder H, Carbon CC (2006) Face-specific configural processing of relational information. Br J Psychol 97:1929.

Leder H, Candrian G, Huber O, Bruce V (2001) Configural features in the context of upright and inverted faces. Perception 30:73-83. 
Maurer D, Grand RL, Mondloch CJ (2002) The many faces of configural processing. Trends Cogn Sci 6:255260.

Maurer D, O'Craven KM, Le Grand R, Mondloch CJ, Springer MV, Lewis TL, Grady CL (2007) Neural correlates of processing facial identity based on features versus their spacing. Neuropsychologia 45:1438-1451.

Mayrhauser L, Bergmann J, Crone J, Kronbichler M (2014) Neural repetition suppression: evidence for perceptual expectation in object-selective regions. Front Hum Neurosci 8: 1662-5161.Mazard A, Schiltz C, Rossion B (2006) Recovery from adaptation to facial identity is larger for upright than inverted faces in the human occipito-temporal cortex. Neuropsychologia 44:912-922.

Nguyen VT, Breakspear M, Cunnington R (2013) Fusing concurrent EEG-fMRI with dynamic causal modeling: Application to effective connectivity during face perception. Neuroimage.

Priebe NJ, Churchland MM, Lisberger SG (2002) Constraints on the source of short-term motion adaptation in macaque area MT. I. the role of input and intrinsic mechanisms. J Neurophysiol 88:354-369.

Rao RP, Ballard DH (1999) Predictive coding in the visual cortex: a functional interpretation of some extraclassical receptive-field effects. Nat Neurosci 2:79-87.

Rhodes G, Brake S, Atkinson AP (1993) What's lost in inverted faces? Cognition 47:25-57.

Rhodes G, Michie PT, Hughes ME, Byatt G (2009) The fusiform face area and occipital face area show sensitivity to spatial relations in faces. Eur J Neurosci 30:721-733.

Riesenhuber M, Jarudi I, Gilad S, Sinha P (2004) Face processing in humans is compatible with a simple shape-based model of vision. Proc Biol Sci 271 Suppl 6:S448-450.

Rosburg T, Ludowig E, Dumpelmann M, Alba-Ferrara L, Urbach H, Elger CE (2010) The effect of face inversion on intracranial and scalp recordings of event-related potentials. Psychophysiology 47:147157.

Rossion B (2008) Picture-plane inversion leads to qualitative changes of face perception. Acta Psychol (Amst) 128:274-289.

Rossion B, Gauthier I (2002) How does the brain process upright and inverted faces? Behav Cogn Neurosci Rev 1:63-75.

Rossion B, Gauthier I, Tarr MJ, Despland P, Bruyer R, Linotte S, Crommelinck M (2000) The N170 occipitotemporal component is delayed and enhanced to inverted faces but not to inverted objects: an electrophysiological account of face-specific processes in the human brain. Neuroreport 11:69-74.

Sawamura H, Orban GA, Vogels R (2006) Selectivity of neuronal adaptation does not match response selectivity: a single-cell study of the FMRI adaptation paradigm. Neuron 49:307-318.

Schacter DL, Dobbins IG, Schnyer DM (2004) Specificity of priming: a cognitive neuroscience perspective. Nat Rev Neurosci 5:853-862.

Schiltz C, Rossion B (2006) Faces are represented holistically in the human occipito-temporal cortex. Neuroimage 32:1385-1394.

Sergent J (1984) An investigation into component and configural processes underlying face perception. $\mathrm{Br} \mathrm{J}$ Psychol 75 ( Pt 2):221-242.

Summerfield C, Wyart V, Johnen VM, de Gardelle V (2011) Human Scalp Electroencephalography Reveals that Repetition Suppression Varies with Expectation. Front Hum Neurosci 5:67.

Summerfield C, Trittschuh EH, Monti JM, Mesulam MM, Egner T (2008) Neural repetition suppression reflects fulfilled perceptual expectations. Nat Neurosci 11:1004-1006.

Tanaka JW, Farah MJ (1993) Parts and wholes in face recognition. QJ Exp Psychol A 46:225-245.

Tanaka JW, Gordon I (2011) Features, configuration and holistic face processing. In Oxford Handbook of Face Perception, 1st Edn, eds A. Calder, G. Rhodes, M. Johnson, and J. Haxby (Oxford: Oxford University Press), 177-1194.

Tanaka JW, Kaiser MD, Bub D, Pierce LJ (2009) Generalized impairment of featural and configural information in the lower region of the face through inversion. Journal of vision 9(8):531.

Weiner KS, Grill-Spector K (2010) Sparsely-distributed organization of face and limb activations in human ventral temporal cortex. Neuroimage 52:1559-1573.

Weiner KS, Grill-Spector K (2013) Neural representations of faces and limbs neighbor in human high-level visual cortex: evidence for a new organization principle. Psychol Res 77:74-97. 
Wiggs CL, Martin A (1998) Properties and mechanisms of perceptual priming. Curr Opin Neurobiol 8:227233.

Xu B, Tanaka JW (2013) Does face inversion qualitatively change face processing: an eye movement study using a face change detection task. J Vis 13.

Yin R (1969). Looking at upside-down faces. J Exp Psychol 81:141-145.

Young AW, Hellawell D, Hay DC (1987) Configurational information in face perception. Perception 16:747759.

Yovel G, Kanwisher N (2004) Face perception: domain specific, not process specific. Neuron 44:889-898.

Yovel G, Kanwisher N (2005) The neural basis of the behavioral face-inversion effect. Curr Biol 15:22562262.

\section{Figure legends}

Fig 1. A. Stimulation parameters and arrangements. An upright repetition trial (RepT), an inverted alternation (AltT) and an upright target trial is illustrated. B. The composition of the repetition and alternation blocks. During a run, RepBs and AltBs were presented randomly, each repeated four times.

Fig 2. A. Whole-brain group results of the functional localizer showing the locations of FFA, OFA and LO. B. The average locations of the pFFA, OFA and LO ROIs as identified in the individual localizer scans. Results are overlayed on top of the average structural image of our subjects.

Fig 3. A. Average peak activation profiles $( \pm S E)$ of the pFFA for AltTs and RepTs separately for each block (2) and orientation (2), but averaged across hemispheres (2). ${ }^{*} \mathrm{p}<0.05,{ }^{*} \mathrm{p}<0.01$ (Fisher's post hoc comparisons). B. The Repetition Suppression Index (RSI, see methods) of the pFFA for the Alternation (AltB) and Repetition (RepB) Blocks separately for the upright and inverted faces, but averaged across hemispheres.

Fig 4. A. Average peak activation profiles $( \pm \mathrm{SE})$ of the OFA for AltTs and Rep'Ts separately for each block (2) and orientation (2), but averaged across hemispheres (2). B. Average peak activation profiles $( \pm \mathrm{SE}$ ) of the LO for AltTs and RepTs separately for each block (2) and orientation (2), but averaged across hemispheres (2). $+\mathrm{p}<0.1,{ }^{*} \mathrm{p}<0.05,{ }^{*} \mathrm{p}<0.01$ (Fisher's post hoc comparisons).

Fig 5. A. Results of the whole-brain group analysis showing the significant activations for the main effects of orientation. B. Results of the voxel-wise whole brain analysis for the interaction of block and trial types separately for UPR and INV faces. $\mathrm{p}<0.0001_{\text {UNCORRECTED }}$; cluster extent of $>20$ voxels. 


\section{Figures}

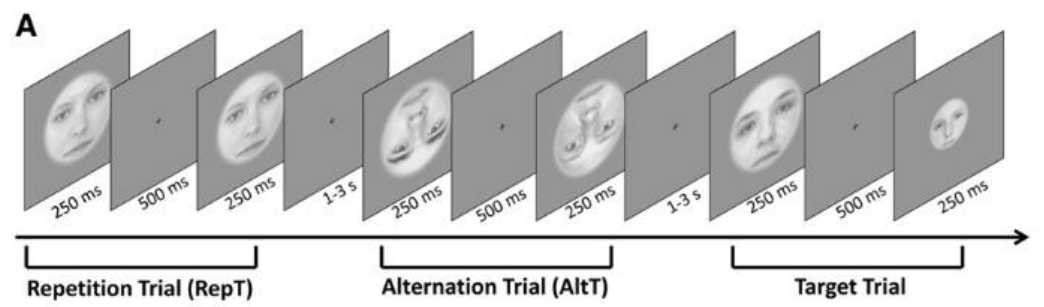

B

Repetition Block (RepB) Alternation Block (AltB)

\begin{tabular}{|l|}
\hline $\mathbf{2 0} \%$ \\
\hline $60 \%$ \\
\hline $\mathbf{2 0} \%$ \\
\hline
\end{tabular}

\begin{tabular}{|l|}
\hline $20 \%$ \\
\hline $20 \%$ \\
\hline $60 \%$ \\
\hline
\end{tabular}

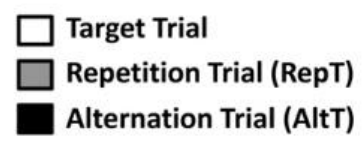

Fig. 1. A. Stimulation parameters and arrangements. An upright repetition trial (RepT), an inverted alternation (AltT) and an upright target trial are illustrated. B. The composition of the repetition and alternation blocks. During a run, RepBs and AltBs were each repeated four times.

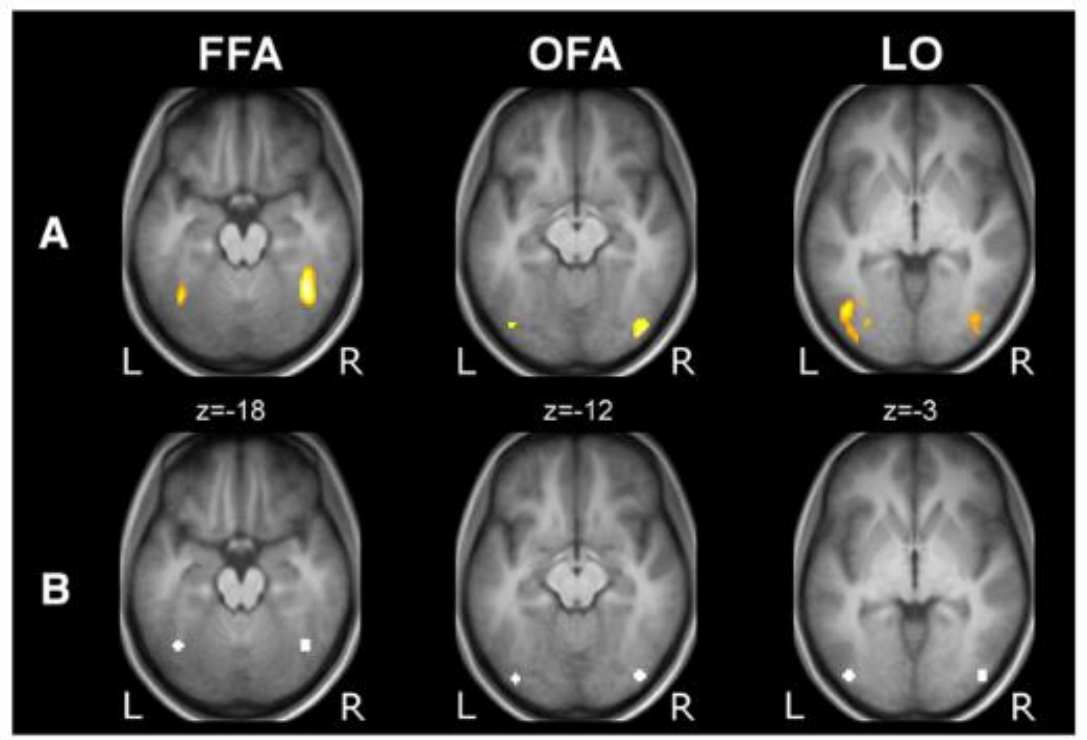

Fig. 2. A. Whole-brain group results of the functional localizer showing the locations of FFA, OFA and LO. B. The average locations of the PFFA, OFA and LO ROIs as identified in the individual localizer scans. Results are overlayed on top of the average structural image of our subjects. 

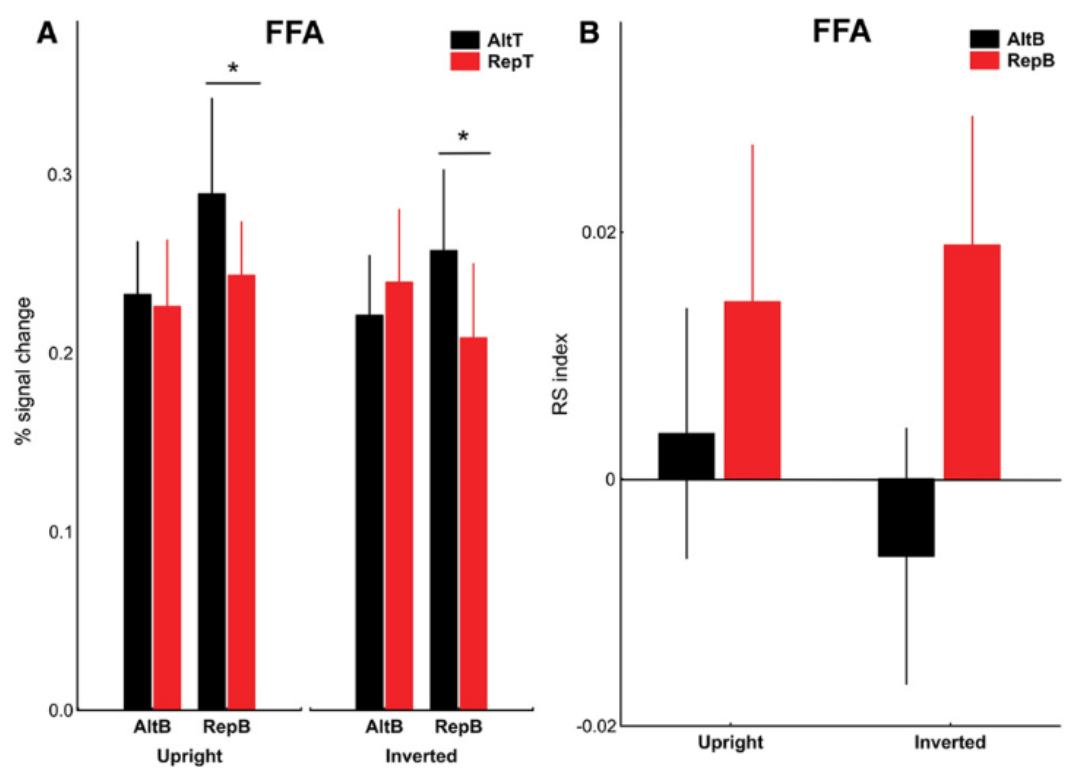

Fig. 3. A. Average peak activation profiles ( \pm SE) of the pFFA for AltTs and RepTs separately for each block (2) and orientation ( 2 ), but averaged across hemispheres ( 2 ). ${ }^{*} p<0.05,{ }^{* *} p<0.01$ (Fisher's post hoc comparisons). B. The Repetition Suppression Index (RSI, see Methods) of the pFFA for the Alternation (AltB) and Repetition (RepB) Blocks separately for the upright and inverted faces, but averaged across hemispheres.

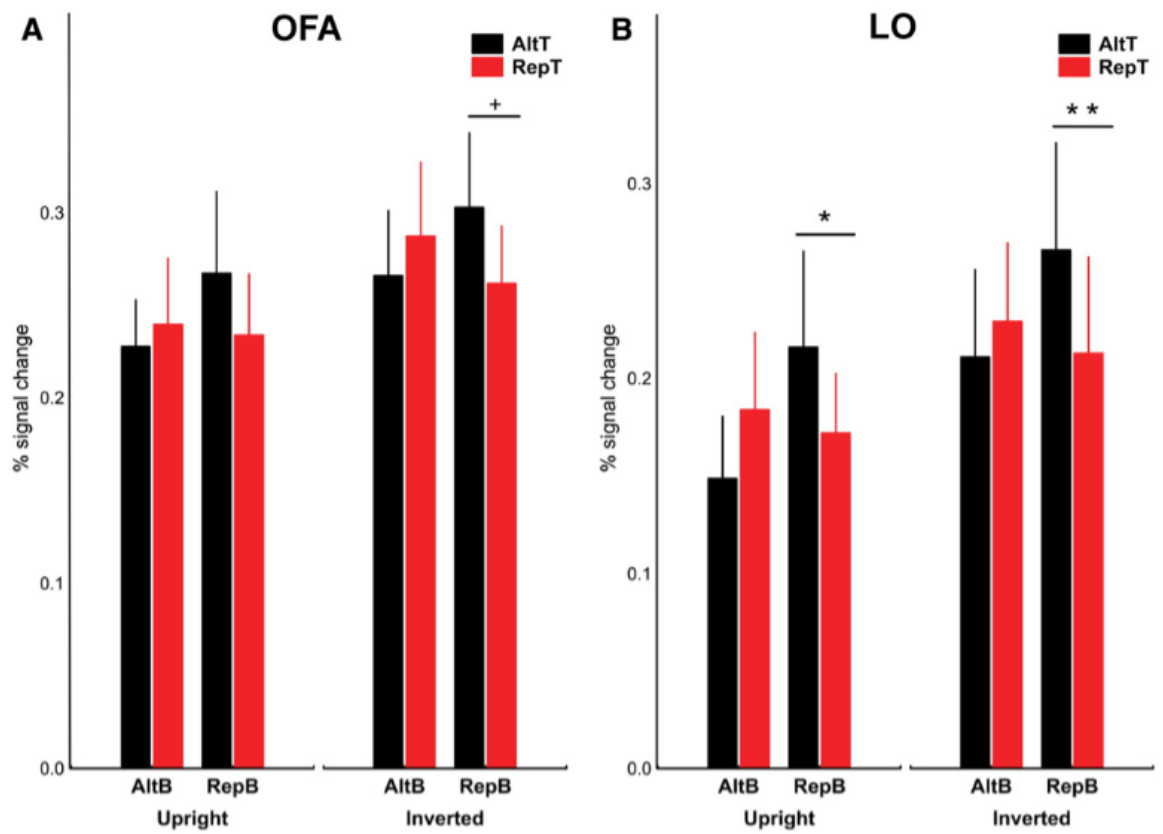

Fig. 4. A. Average peak activation profiles ( \pm SE) of the OFA for AltTs and RepTs separately for each block (2) and orientation (2), but averaged across hemispheres (2). B. Average peak activation profiles ( \pm SE) of the LO for AltTs and RepTs separately for each block (2) and orientation (2), but averaged across hemispheres $(2)$. $+p<0.1$, " $p<0.05$, " $p<0.01$ (Fisher's post hoc comparisons). 


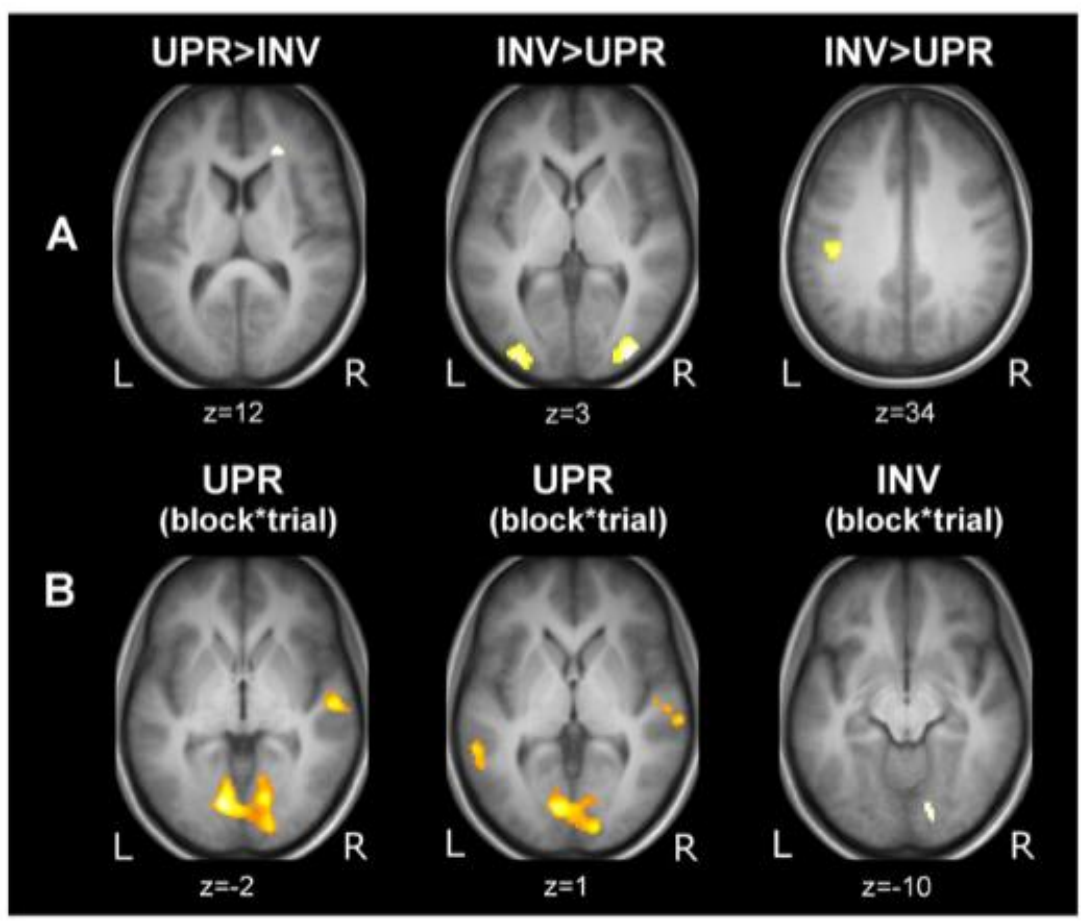

Fig. 5. A. Results of the whole-brain group analysis showing the significant activations for the main effects of orientation. B. Results of the voxel-wise whole brain analysis for the interaction of block and trial types separately for UPR and INV faces. $p<0.0001_{\text {UNCORRECTED; }}$ cluster extent of $>20$ voxels. 